\title{
Modeling Native INTELligenCE SEMANTICS FOR INDIGENOUS SELECTION OF WEB SERVICES USING SLAKY COMPOSER
}

\author{
P. Sandhya ${ }^{1}$ and Dr. M. Lakshmi ${ }^{2}$ \\ ${ }^{1}$ Research Scholar, Department of Information Technology, Sathyabama University, \\ Chennai,Tamil Nadu, India \\ catherinesandhya@gmail.com \\ ${ }^{2}$ Professor and Head, Department of Computer Science and Engg,, Sathyabama \\ University, Chennai,Tamil Nadu, India \\ laks@icads.co.in
}

\begin{abstract}
Automatic web service composition is defined as the construction of virtual enterprises as complex services on demand. In the current research scenario choosing business service partners for composition on the fly is usually determined with user-centric metrics and deficient of provider's business specific metrics. Automatic web service composition in today's real world business circumstances is more of a toy model due to the lack of service provider collaboration metrics [1]. SLAKY System is a new model for selection of business service partners by adding service provider metrics to user centric metrics enabling strategic and realistic selection of services for composition. SLAKY System selects services automatically considering the vision, time planning, environmental context, user adoption, usage policies, trust management, risk management, market scenario, native intelligence, and competitive profit management as service provider collaboration metrics in addition to functionality satisfaction metrics for client's requirements. The time planning metric was designed using opus deviser algorithm [2] and profit management using SLAKY BWG algorithm [3]. In this paper we focus on strategic selection of semantic services based on the metric of native intelligence. Howard Gardner, psychology professor, Harvard University, defines intelligence as an ability to solve problems, or to create products, that are valued within one or more cultural settings. Richard Heck, Professor of Natural Theology, Brown University, in his work states that native intelligence is primarily influenced by racial and cultural differences. User preferences in any sector intend to have native influence. For example the affinity towards entertainment services for Indian Premier League or low cost car booking service for TATA Nano is more in India than America. Moreover decision made by a decision maker is motivated by native intelligence in addition to Intelligence Quotient and is valued highly in native grounds. For example the selection of a matrimonial service totally relies on native aspects. Native Intelligence is also a significant metric to improve time-to-market and quality in the launch of any product. Considering the significance of native intelligence in strategic selection we layer native intelligence descriptions for realistic selection of semantic web services on the fly. OWL-S upper ontology that describes semantics of services for automation of service discovery, selection, composition, orchestration and invocation lacks entities for semantic modeling of native intelligence. In this paper we propose an extended OWL-S ontology which augments where, why and who native status descriptions of a service apart from the existing what (ServiceProfile sub-ontology) and how (ServiceModel, ServiceGrounding sub-ontology) semantics. The why, where and who semantics are newly augmented as ServiceParameters of Profile sub-ontology using sParameter property. We also propose Naavi algorithm that reasons with common sense knowledge for selection of the web service that is highly valuable in a native context using native description in extended $O W L-S$.
\end{abstract}




\section{KEYWORDS}

SLAKY Composer, OWL-S, Extended OWL-S, Native Intelligence, Backward Chaining;

\section{INTRODUCTION}

Today conventional business models have been greatly revamped through internet based enterprises. New business models based on web services like freemium have emerged rendering huge profit. Atomic services are aggregated to offer complex services through web service composition. Web services integrate different verticals through composition and create virtual enterprises on the web. The process of composition can be manually knit at design time using workflow languages like Business Process Execution Language. Static composition has less flexibility as business partners are fixed. Static composition does not provide choice over selection of services that actually satisfy the requirement of the user. Automatic composition selects services on the fly at runtime such that the services are more specific to user requirements and satisfy QOS constraints. To automate composition web services are annotated with their domain and functional specification enabling agents to automate. The semantic annotations are based on OWL-S and domain ontology. There are several works that enable automated selection more specific to user's context specification and QOS specification. However much of the work in service composition concentrates purely on user centric metrics and does not focus on real world business collaboration metrics. SLAKY is a service composition architecture based on real world business collaboration metrics. SLAKY architecture allows choosing service partners based on both user and provider metrics making service composition more realistic. The time planning metric was designed using opus deviser algorithm and profit management using SLAKY BWG algorithm. In this paper we have proposed composition based on the metric of native intelligence. We have also proposed Naavi algorithm to select services based on native intelligence. We have also proposed an extended semantic modeling of web services by extending OWL-S with ServiceParameter for native intelligence and appropriate domain ontology.

\section{RELATED WORKS}

There are several works done in the field of web service composition. In 2010 effort-oriented classification matrix was proposed for web service classification [4]. Semantic web service composition algorithm was also proposed using QOS ontology [5]. Research on semantic based web service composition was done by Cao in 2010 [6]. QOS aware web service composition based on pre and post conditions were also proposed in 2010 [7]. Dynamic web service composition based on QOS was proposed [8]. In [9] semantic based mashups was designed using composite applications. In 2011 automated workflow composition for semantic web services was proposed [10]. Though there are several semantic web service composition techniques all of them focus on QOS. The real time dynamics of business environment and metrics of a business oriented decision maker are not considered. In this paper we newly consider native intelligence as a metric apart from other OWL-S metrics.

\section{Need for Programming Native Intelligence in Business Models BASED ON WEb SERVICE COMPOSITION}

Howard Gardner, psychology professor, Harvard University, defines intelligence as an ability to solve problems, or to create products, that are valued within one or more cultural settings [11]. Oxford dictionary defines Intelligence Quotient as an entity primarily influenced by racial and 
cultural differences [12]. Sanjeev Bickhchandani, founder, Naukri.com, quotes that the success of his company is that they adopted a common sense approach [13]. Common sense is the outcome of native intelligence [14]. The choice of any service must functionally satisfy user requirements. User preferences in any sector intend to have native influence. For example the affinity towards entertainment services for Indian Premier League or low cost car booking service for TATA Nano is more in India than America. Moreover decision made by a decision maker is motivated by native intelligence in addition to Intelligence Quotient and is valued highly in native grounds [15]. For example the selection of a matrimonial service totally relies on native aspects. We therefore propose to augment native intelligence metric for user requirement and service provider collaboration. Applying native intelligence by service provider promotes selection of appropriate services in a native context. A skilled administrator would apply a certain degree of native intelligence to make decision. Such decisions would offer strategic solutions for enterprises. Most of the relevant works on composition algorithms are more focused on offering apt services to clients. However the vision of enterprises is to make success. Strategic solution by managers should offer good product to client and promote the goals of the enterprise. The strategic solutions are partly dependent on native intelligence. In SLAKY architecture we integrate native intelligence as a service provider metric for selection of services for composition apart from client's specification.

\section{SLAKY ARCHITECTURE}

\begin{tabular}{|l|c|}
\hline \multicolumn{3}{|c|}{ EXECUTION OF COMPOSITION } \\
\hline \multicolumn{3}{|c|}{ STRATEGIC COMPOSITION OF SELECTED SERVICES THAT MEETS USER, } \\
PROVIDER AND AGENT DYNAMICS
\end{tabular}

Figure. 1. SLAKY Collaboration Stack

SLAKY is a realistic model for choosing business service partners considering service partner collaboration metrics including vision, time planning, environmental context, user adoption, usage policies, trust management, risk management, market scenario, native intelligence and competitive profit management of service partners apart from functionality satisfaction for 
client's requirements. We have proposed SLAKY architecture because the choice of a service partner should not only satisfy end user's requirements but also meet real world business objectives of the composite application provider. SLAKY composer chooses services such that they give high profit computed by profit management module using SLAKY BWG algorithm. The success of any product is reflected by the adoption of the product by user and industry. User adoption of products is dependent on native intelligence. The success story of TencentQQ winning over MSN was contributed by the adoption of native intelligence. The native Chinese beheviour is to trade-off a certain level of privacy protection in virtual community to gain more chances of inviting strangers [16]. TencentQQ dominates local instance message market in China and has gained attention of international IT role players by embracing native intelligence. In this paper we focus on programming native intelligence using Naavi algorithm. OWL-S upper ontology which describes the semantics for automation web service composition lacks concepts that captures native intelligence. Therefore in this paper we extend OWL-S to include certain ServiceParameters to define native status of any service and native user beheviour so that Naavi algorithm would apply native intelligence to evaluate and select the service.

\section{Automation of Web Service Composition Using OWL-S SEMANTIC DESCRIPTION OF SERVICES}

WSDL describes services at syntactic level requiring human intervention for composition of services [17]. To automate composition meaningfully the semantics of service functionality have to be described using ontology. To obtain service descriptions there are two kinds of ontologies namely Generic web service ontology like OWL-S to specify input, output, precondition and effect and a domain ontology to specify web service domain knowledge such as service parameters and domain ontology. The generic web service ontology has four subontologies namely Profile, Process, Grounding and Service. Profile ontology specifies what a service does, Process ontology specifies how the service works and Grounding ontology specifies how the service is implemented. The Service ontology links ServiceProfile, ServiceProcess and ServiceGrounding which are further specialized as sub-concepts in Profile, Process and Grounding ontology.

The profile ontology specifies what the service does as a functionality offered by the service, semantic type of inputs outputs precondition effect, details of service provider, and several service parameters like quality rating and geographic radius. Service discovery is based on this description. For each of the functionality of a specific service there is a profile. The hasProc relation specifies the association of each Profile instance with the process it describes.

Process ontology describes the internal process models of complex services. The process model is used to automatically compose the web services. The Process ontology has a concept called ProcessModel which describes a single Process. The process can be atomic, simple or composite with or without control constructs. The IOPE of Profile and Process are linked by refersTo property.

Grounding ontology describes the vocabulary to link conceptual description of service specified by Profile and Process to actual implementation details. The implementation details include network protocols, message etc. The WSDLGrounding contains WSDL description that treats AtomicProcess as a WSDL operation. Therefore the inputs and outputs of AtomicProcess are mapped to corresponding messagepart in the input message and output message respectively of WSDL operation. The Grounding ontology specializes ServiceGrounding as a WSDLGrounding. The type of WSDL messagepart is specified as a OWL-S parameter. Each 
WsdlAtomicProcessGrounding elements of WSDLGrounding grounds an atomic process in ProcessMode

The semantic description of service is offered by Profile and Process ontology. WSDL offers the syntactic descriptions of the service. The mapping between syntactic and semantic descriptions is given by Grounding ontology.

To enrich OWL-S ontology with domain knowledge domain descriptions are specified in domain ontology. The domain ontology is based on OWL and contains a DataStructure and Functionality hierarchy. The functionality hierarchy supports better discovery than a normal key-word based discovery.

Though OWL-S captures what and how semantics of any service it fails to capture native descriptions of where, why and who semantics of a service. We extend OWL-S generic ontology to augment definitions of where, why and who ServiceParameters for natively good service selection from both provider and user perspective. Section 5 discusses the inclusion of native constructs in extended OWL-S.

\section{EXTENDED OWL-S WITH SEMANTIC DESCRIPTION FOR NATIVE INTELLIGENCE}

WSDL describes services at syntactic level requiring human intervention for composition of services. To automate composition meaningfully the semantics of service functionality

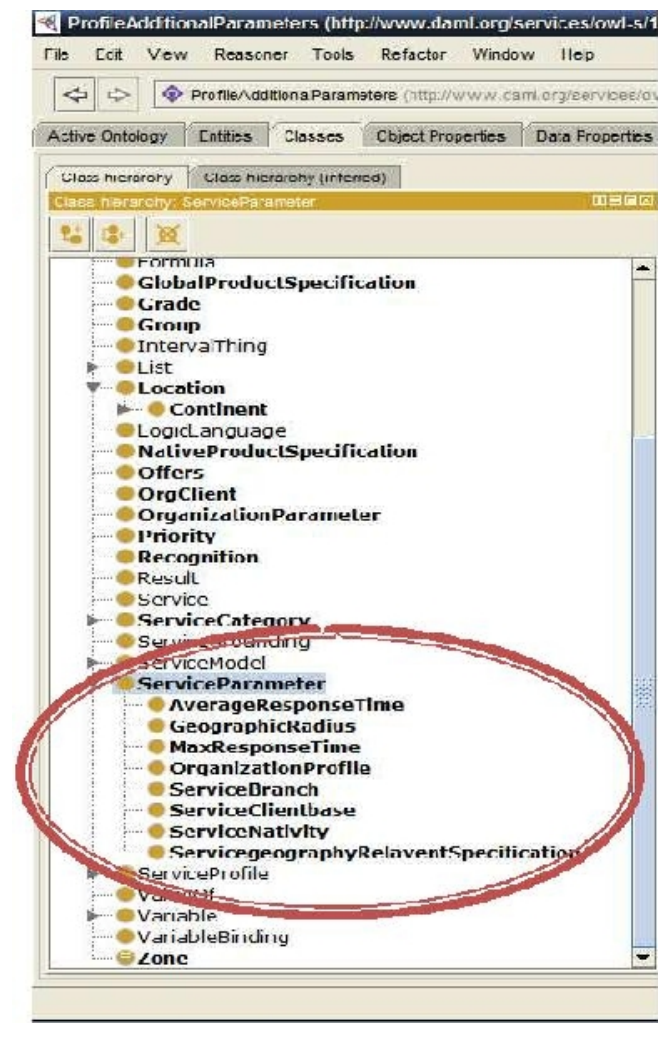

Figure. 2. ServiceParameters for Augmenting Native Intelligence 
International Journal on Cloud Computing: Services and Architecture (IJCCSA),Vol.2, No.5, October 2012

The native intelligence semantics are augmented as ServiceParameter subconcepts namely OrganizationProfile to capture who semantics, ServiceBranch, ServiceClientbase, ServiceNativity to capture where semantics and ServiceGeographyRelaventSpecification to capture why semantics.

\subsection{Capturing Where Semantics of Services}

The choice of selection of a service is influenced by the native origin of the service, the branches where the service is provided and the geography of the clients. The concepts ServiceNativity, ServiceClientbase and ServiceBranch has range of individuals of Location concept.

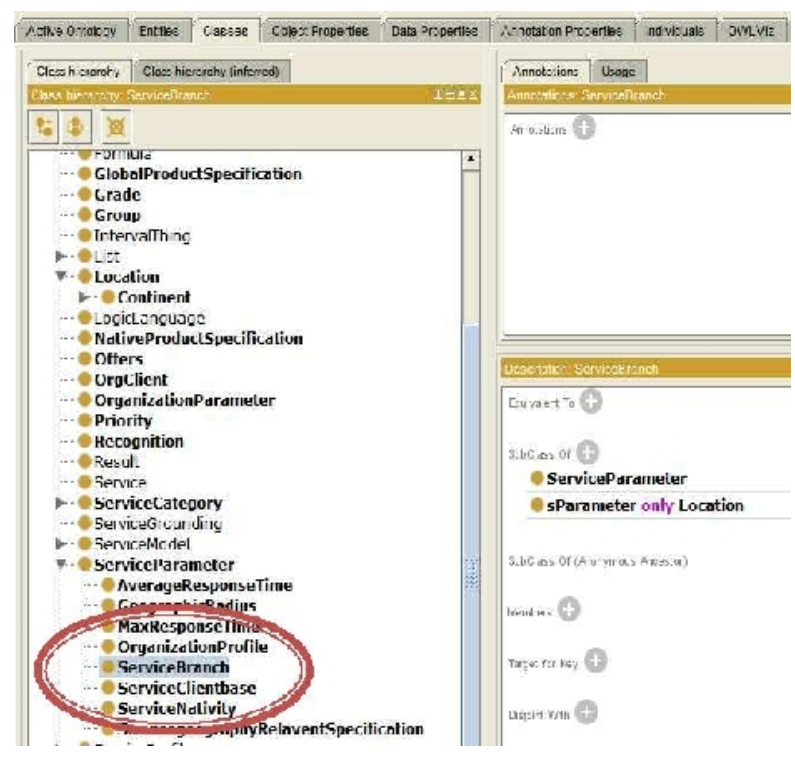

Figure. 3. ServiceBranch, ServiceClientbase, ServiceNativity have individuals of Location Concept

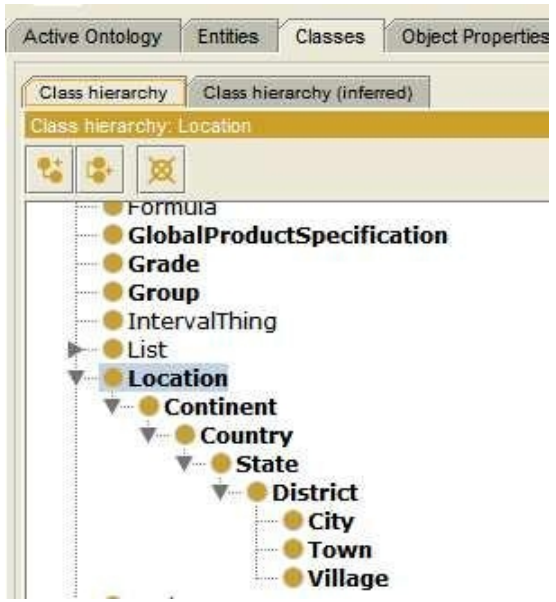

Figure. 4. Location Concept 


\subsection{Capturing Why Semantics of Services}

ServiceGeographyRelevantSpecification captures the GlobalProductSpecification, LocalProductSpecification and CostSpecification semantics of the service. A composing agent can evaluate why to choose the service in terms of its status in global and local scenario. The service can have different preferences based on its geography. The features of the service relative to global and local scenario have to be described. For example a certain service can be a pioneering service locally but a conventional service globally. Certain services can be described for local speciality. A typical example is that naukri is the first job search site in India. A search for kancheepuram silk sarees would be best in South India at Kancheepuram for authentic products. Kancheepuram silk sarees are typical native creations from Tamil Nadu. The cost specification is also influenced by native metrics. Special price offers would be offered during native festive seasons.

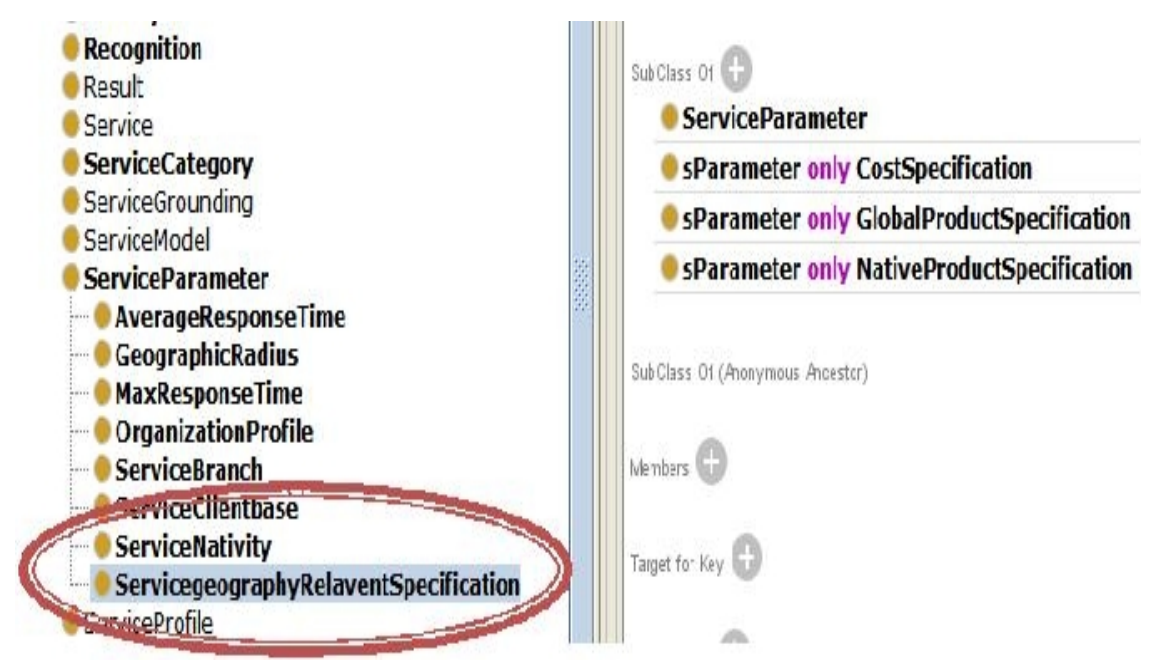

Figure. 6. Why Semantic Descriptions
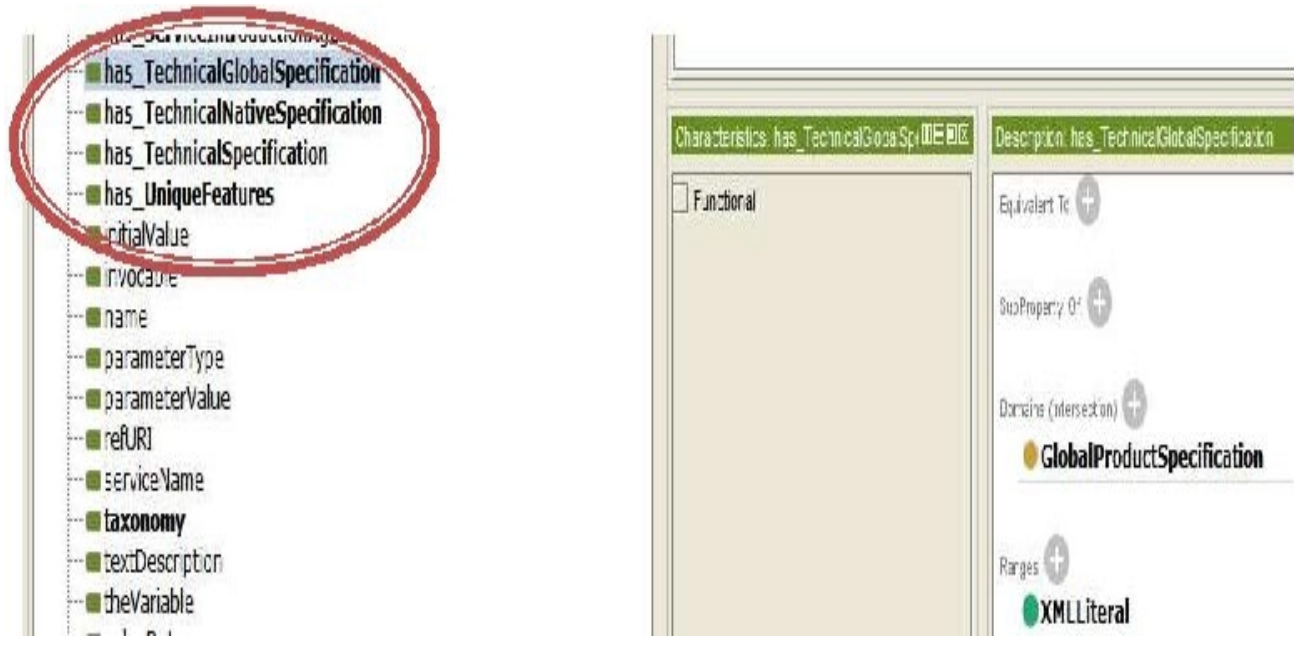

Figure. 7. Data Properties with Domain and Range Specification for Why Semantic Description 


\subsection{Capturing Who Semantics of Services}

In a real world scenario choosing a business service partner is based on the organization group affiliation, collaborators, recognitions, administrator, grade, kind of other clients, client priority offered, age of the organization, age of the brand, standard conforming to, etc. These semantic descriptions describe who exactly the service provider is. We capture these semantics in OrganizationProfile concept that has range of individuals in OrganizationParameter.

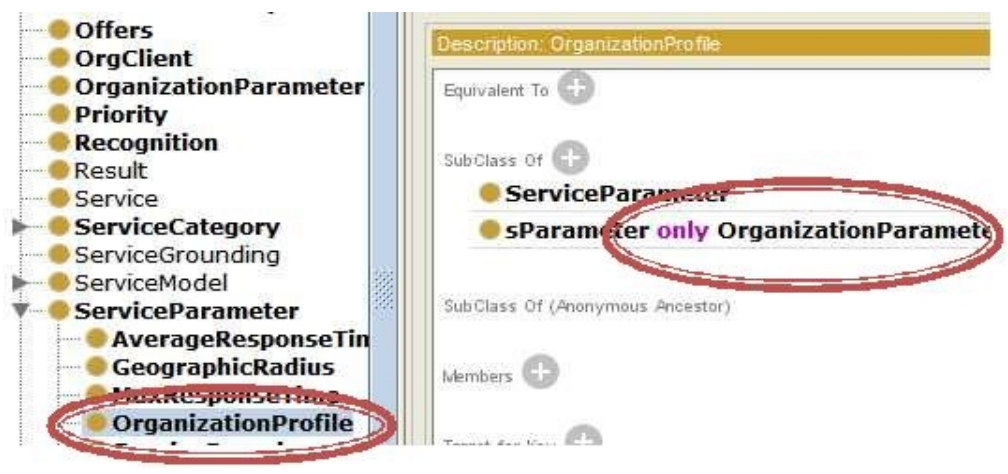

Figure. 8. OrganizationParameter Captures who Semantics of the Service Provider

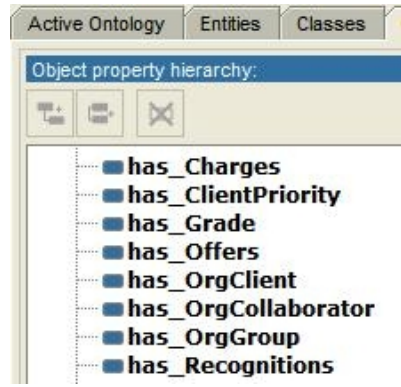

Figure. 9. Object Properties - OrganizationParameter

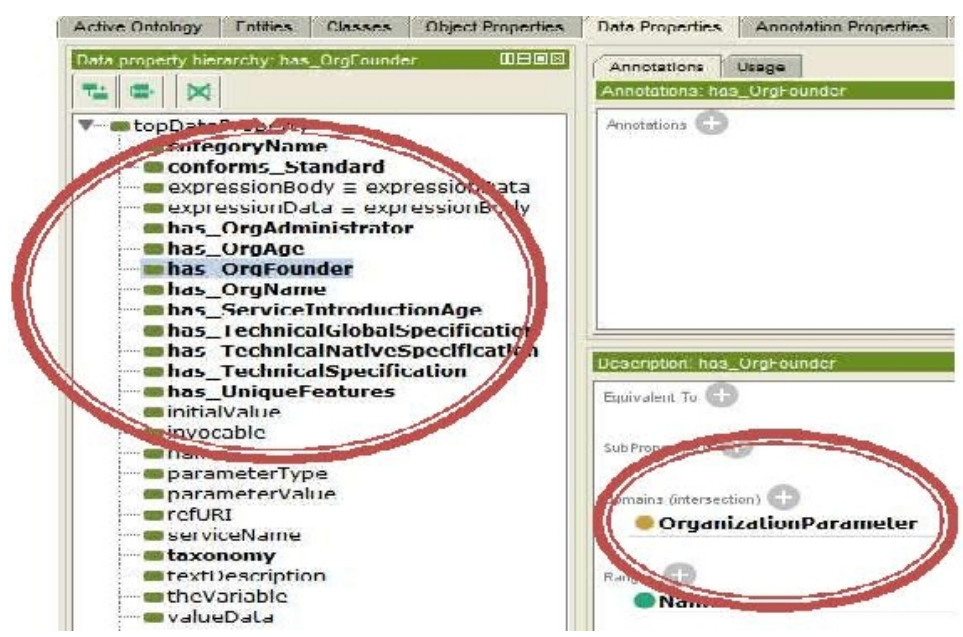

Figure. 10. Data Properties with Domain and Range - OrganizationParameter 


\section{ANNOTATION View of SLAKY NATIVE INTELligenCE Module}

SLAKY composer considers native intelligence as a metric to select indigenous services on the fly. The knowledge base of the composer system is populated with Schema axioms in TBox and data related axioms in ABox [18].

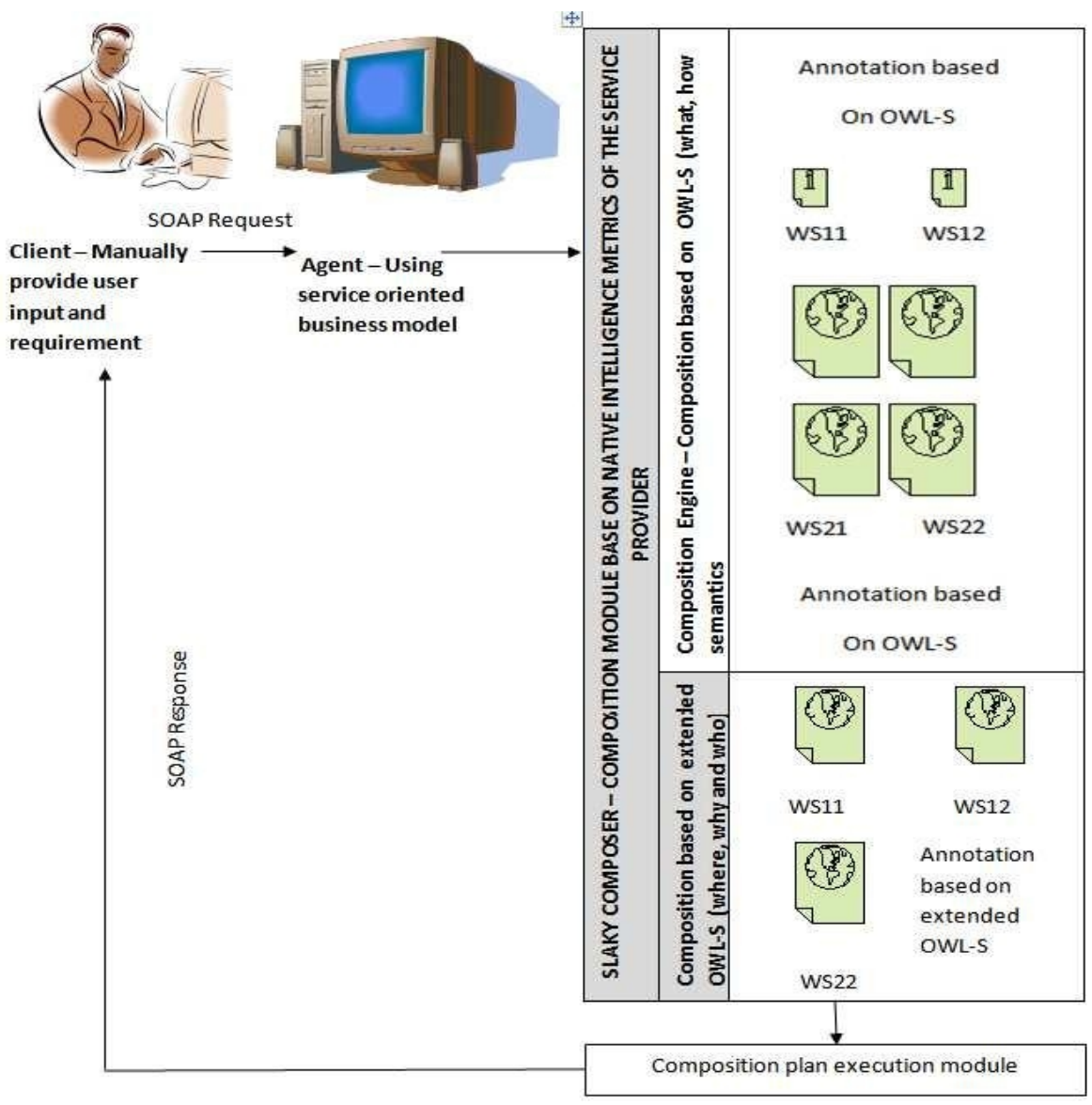

Figure. 11. SLAKY Annotation View

SLAKY system consists of user interface, knowledge base and an inference engine. The composer module of the system chooses an appropriate service yielding native metric enriched services. In fact most of the decisions made in enterprises are primarily influenced by native aspects.

The user interface is designed to capture functional and native metrics. A typical scenario considered here is an example of semantic selection of matrimonial service. The success of a matrimonial service absolutely depends on native aspects. When Input and preconditions are set in accordance to profile ontology we should extend the native metrics as well. Here the inputs are religion, age, gender, country, qualification and salary. The pre condition is set as unmarried or divorced status or widow or widower. On the other hand there are native specific metrics including caste, age, mother tongue, partner preference, salary, etc. 
International Journal on Cloud Computing: Services and Architecture (IJCCSA),Vol.2, No.5, October 2012
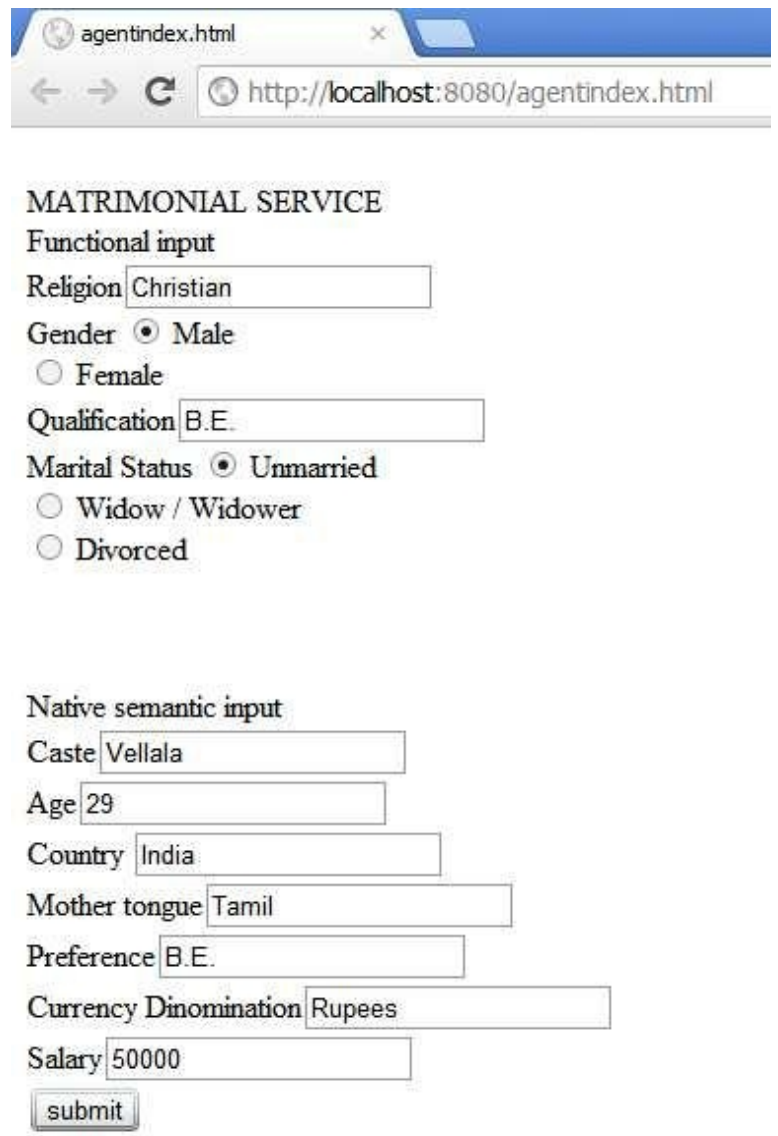

Figure. 12. User Interface

Based on semantic annotations using OWL-S upper ontology matrimony services are selected on the fly. In SLAKY system we have augmented native specific semantics over OWL-S annotation layer to synthesize indigenous services. The native semantics augmented to OWL-S ServiceParameter. ServiceParameter code is presented below.

package sandhya;

import java.util.Collection;

import org.protege.owl.codegeneration.WrappedIndividual; import

org.semanticweb.owlapi.model.OWLNamedIndividual; import org.semanticweb.owlapi.model.OWLOntology;

public interface ServiceParameter extends WrappedIndividual

\{

OWLNamedIndividual getOwlIndividual(); OWLOntology getOwlOntology();

void delete();

\} 


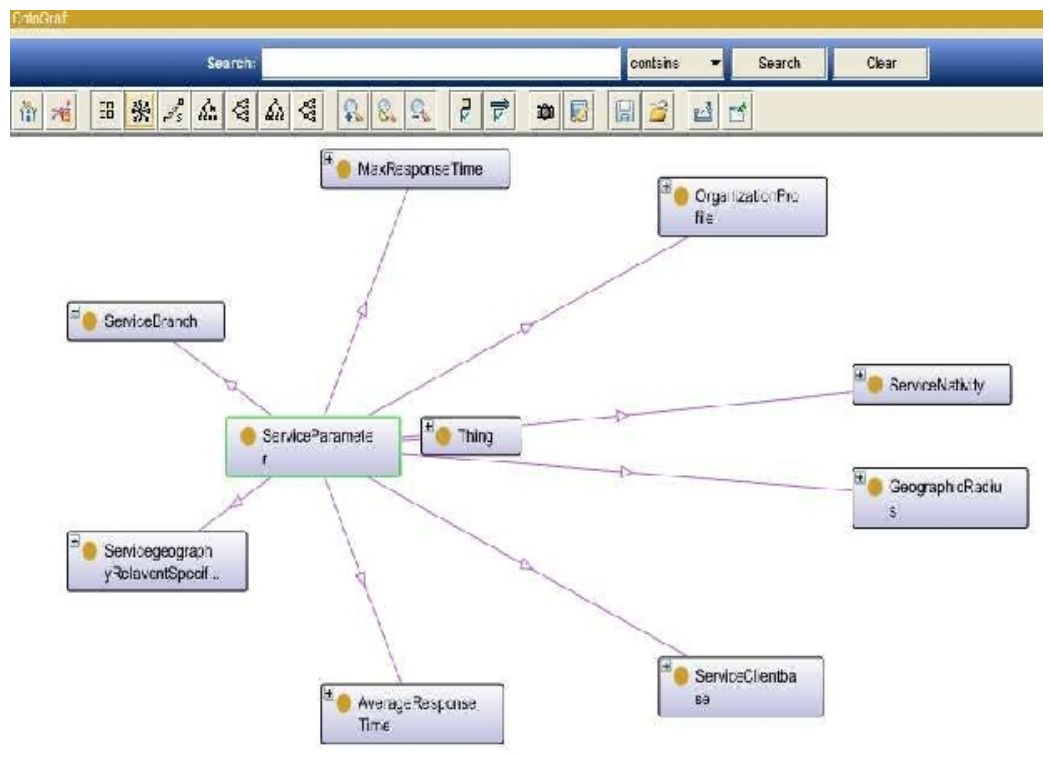

Figure. 13. Overview of ServiceParameter

\subsection{Where Semantics - Example}

ABox axioms (Concept assertion) -

Continent(Asia)

Country(India)

State(TamilNadu)

City(Chennai)

ABox axioms (Role assertion) -

hasCountryNeighbour(India,Japan)

Asia, India, TamilNadu and Chennai are individuals of respective concepts.

Individual India hasCountryNeighbour individual Japan where hasCountryNeighbour is a role.

The role hasCountryNeighbour is reflexive.

TBox axioms (Term assertion - Concept inclusion) - ServiceClientBase 드 Location

ServiceBranches $\sqsubseteq$ Location

ServiceNativity 드 Location

RBox axioms (Role inclusion) or complex Role composition hasCountry o hasState o hasDistrict ㄷ hasContinent Nominals -

Continent $\equiv\{$ Asia $\} \sqcup\{$ Europe $\} \sqcup\{$ Africa $\} \sqcup\{$ America $\}$ A Auatralia $\} \sqcup\{$ Antarctica $\}$ Continent concept thus has 6 continents. 


\subsection{Why Semantics - Example}

TBox axiom (Concept inclusion) -

GlobalProductSpecification 드 ServiceGeographyRelevantSpecification

LocalProductSpecification드 ServiceGeographyRelevantSpecification

CostSpecification 드 ServiceGeographyRelevantSpecification

ABox axioms (Role assertion) -

hasLocalProductSpecification(BharathBenz,First_Lower_cost_model_in_India)

hasCostSpecification(BharathBenz,400000)

hasUniqueFeature(BharathBenz,engine_on_Indian_standards)

BharathBenz is a low cost model launched mainly focussing Indian customers. On the global scale heavy duty vehicles of Benz are sophisticated models undoubtedly. However BharathBenz is the First_Lower_cost_model_in__India and costs Rs. 400000 represented as a XML Literals. The specifications of BharathBenz are unique to Indian norms.

\subsection{Who Semantics - Example}

OrganizationParameter has OrganizationProfile that helps the decision maker to evaluate the selection of the service based on the status of the provider. All the roles both data and object properties are set as disjoint.

ABox axioms (Role assertion) has_orgFounder(Apple,Steve_Jobs)

\section{IMPLEMENTATION}

User interface is designed to get user inputs. User inputs will have both functional and native specific inputs. The IOPE details are given in accordance to OWL-S upper ontology. The OWL-S extended ontology was extended from OWL-S upper ontology using protégé_3.4.8 and OWL-S plugin. Service selection in SLAKY with native intelligence is illustrated with an example.

Consider that a client needs to find matrimonial services for Christian religion and community as vellala with Tamil as their mother tongue. As a search for Christian matrimony there are services for American Christian services, European Christian matrimony services and Indian Christian. Though the client needs a Christian matrimony service his native behavior would suggest selecting Indian matrimony service. The client's mother tongue being Tamil would result in a smaller granular search in Tamilnadu. The search could be extended to neighboring states as they all belong to the same ethnic groups.

MatrimonyPreference $\equiv$

$\exists$ ᄏhas_country.Country $\Pi$

$\exists$ Gas_state.State $\Pi \exists$ has_mother_tongue.MontherTongue

ExtendedPreference $\equiv \exists$ has.StateNeighbour.StateNeighbour

The why semantics describes why a particular service should be selected. The

ServiceGeographyRelevantSpecification defines local and global product specification with cost metrics. The functionality provided for native clients can be more than global clients or viceversa. Typically during specific auspicious months in India there is a high demand of matrimonial services. This service has no impact globally. 


\section{MatrimonyPreference $\equiv$}

LocalProductSpecification ("Season offers")

The who semantics describes the success history of an enterprise. When a service needs to collaborate with other service there are certain metrics to be evaluated.

has_OrgName(ChristianMatrimonyService, BarathMatrimony)

The set of axioms form the knowledge base. The ontologies were checked for correctness, consistency, minimally redundant and meaningfulness using Fact++ reasoner. To access the ontology Jena API was used.

The inference engine derives logical conclusions from the knowledge base. The inference engine infers through backward chaining.

STEP 1: The initial goal is set as selection of a matrimonial service.

STEP 2: Check for the matches of native goals (sub goals) from the user and goals in the rules of the knowledgebase.

STEP 3: A recursive procedure is done so that all the conditions are checked.

STEP 4: If any condition is true or false repeat the recursive call.

STEP 5: When recursion is over if all conditions are true then true is returned and exited. In case the condition is false then exit.

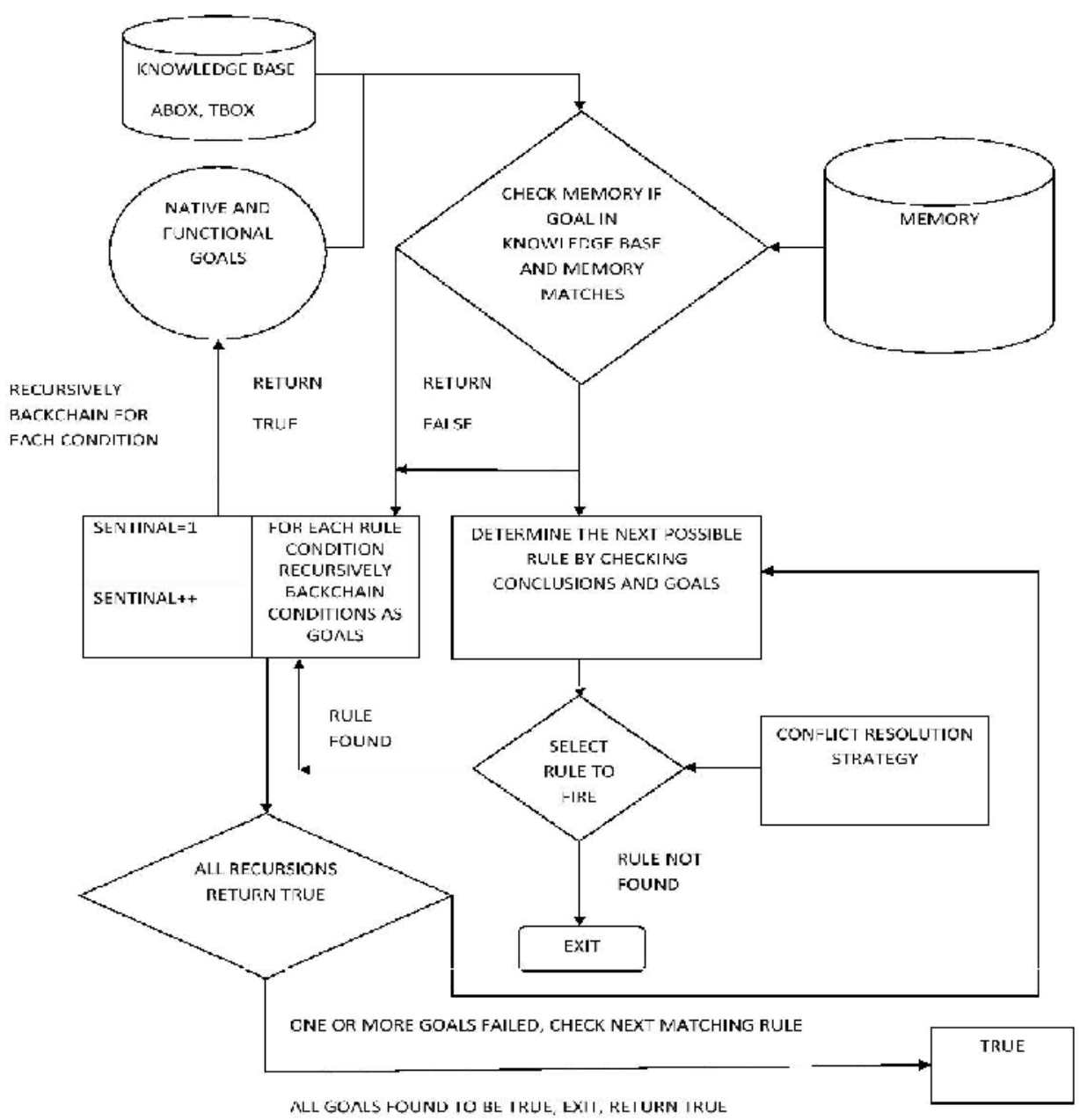

Fig. 14. Naavi - Backward Chaining 


\section{RESULTS AND DISCUSSION}

User interface is designed to get user inputs. User inputs will have both functional and native specific inputs. The IOPE details are given in accordance to OWL-S upper ontology. The OWL-S extended

Four matrimony services were created and annotated based on extended OWL-S. On execution based on functional requirement all the four services were chosen. Asper the functional and native requirements the goals were matched with Abox and Tbox axioms in the knowledgebase. The inference is made by backward chaining using Naavi algorithm for inputs in Fig. 12. Each subgoal is tested.

Table. 1 Annotations

\begin{tabular}{|l|l|l|l|l|}
\hline SERVICE NAME & COUNTRY & RELIGION & $\begin{array}{l}\text { MOTHER } \\
\text { TONGUE }\end{array}$ & CASTE \\
\hline $\begin{array}{l}\text { KALYANAMALAI } \\
\text { MATRIMONY }\end{array}$ & INDIA & HINDU & TAMIL & ANY \\
\hline $\begin{array}{l}\text { BARATH } \\
\text { MATRIMONY }\end{array}$ & INDIA & $\begin{array}{l}\text { CHRISTIAN, } \\
\text { HINDU, } \\
\text { MUSLIM }\end{array}$ & $\begin{array}{l}\text { ANY } \\
\text { NATIVE } \\
\text { LANGUAGE }\end{array}$ & ANY \\
\hline $\begin{array}{l}\text { XYZCASTE } \\
\text { MATRIMONY }\end{array}$ & INDIA & $\begin{array}{l}\text { ABC } \\
\text { RELIGION }\end{array}$ & TAMIL & XYZ \\
\hline $\begin{array}{l}\text { LONDON } \\
\text { MATRIMONY }\end{array}$ & BRITAN & CHRISTIAN & ENGLISH & NILL \\
\hline
\end{tabular}

For the functional inputs given above there are four matrimonial services providing the Christian matrimony functionality. By Naavi however Bharath matrimony is selected due to native specification as shown in Figure. 15.

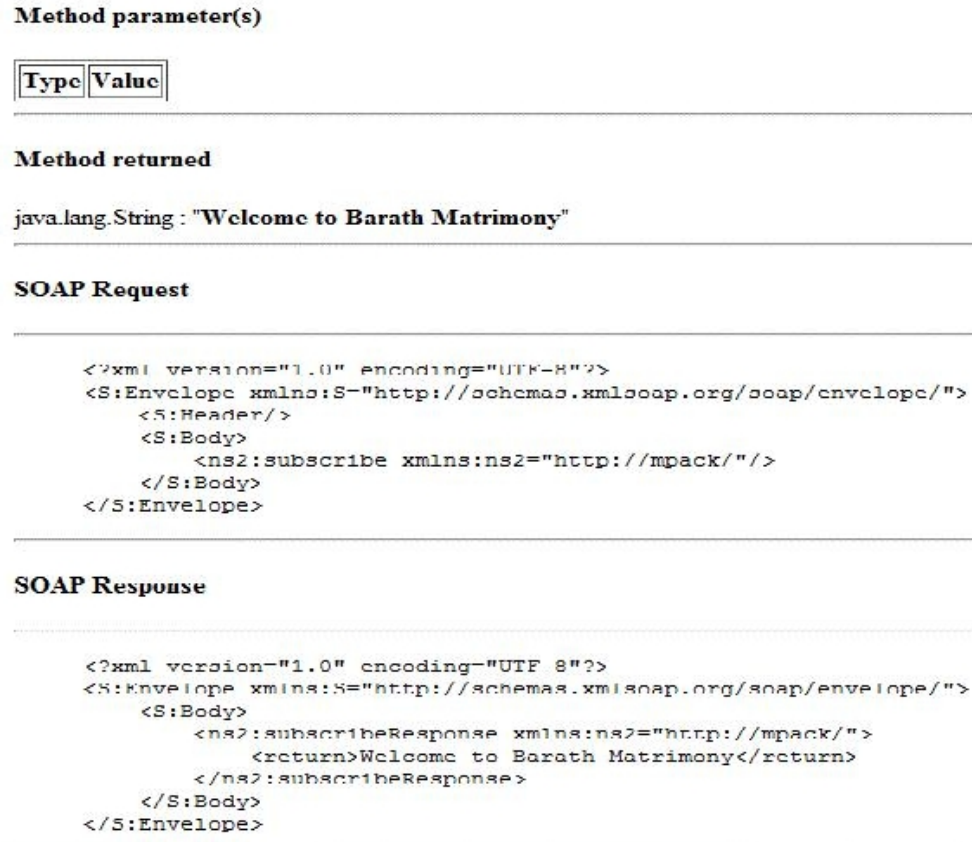

SOAP Respunse

Figure. 15. Service Selection based on extended OWL-S 


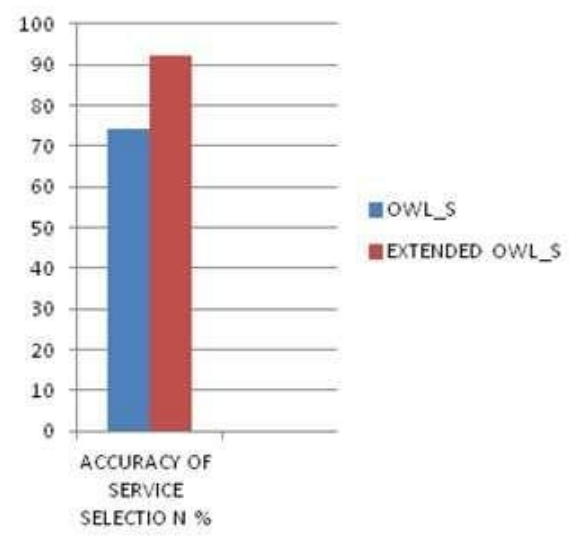

Figure. 16. Evaluation of extended OWL-S

\section{CONClusion AND Future Enhancement}

In this paper we have annotated services with OWL-S native intelligence metrics. In the paper we also augment where, why and who semantics apart from what and how. Decision making is best done with a notion of native intelligence. All other semantic web service business models lack native intelligence. Augmenting native semantics brings natively good service selection. As discussed selection of a Christian matrimony service is provided by most countries. However the native Indian Christian matrimony service is the preferred service for an Indian Christian. Modeling native intelligence for every ethnic group is still a herculean task. In a developing country like India the influence of western culture has brought a major change. Cultures influence each other. As a future enhancement modeling the influence of other culture on native intelligence should be probed. In the current scenario where service composition has not yet been used as standardized business model, realistic metrics like native intelligence will pave the way.

\section{REFERENCES}

[1] Nikolay Mehandjiev, Freddy Lecu'e, Usman Wajid, Provider-Composer Negotiations for Semantic Robustness in Service Compositions, Proceedings of the 7 th International Joint Conference on Service-Oriented Computing, ICSOC-ServiceWave '09, ISBN: 978-3-642-10382-7, pg. 205-220, Springer-Verlag Berlin, Heidelberg @2009.

[2] P. Sandhya, Dr. M. Lakshmi,, A Novel Approach for Realizing Business Agility through Temporally Planned Automatic Web Service Composition Using Network Analysis", Worldcomp '11, (SWWS'11), LasVegas, USA on July 18-21, 2011, CSREA Press, U.S.A, ISBN: 1-60132-202-X, Pg No: $24-31$

[3] P. Sandhya, Dr. M. Lakshmi, Strategic Composition of semantic web services using SLAKY composer, WEST-2012, Advances in Intelligent Systems and computing, July 13-15, 2012, pg. 411421, Adnavces in computing and information technology, Springer, ISBN978-3-642-31599-2, ISSN2194-5357, Available online Springerlink.com

[4] Zheng Li, Liam O’ Brien, Jacky Keung, Xiwei Xu, Effort-Oriented Classification Matrix of Web Service Composition, 2010 Fifth IEEE International Conference on Internet and Web Applications and Services,pg.357-362.

[5] Hai Van, Wang Zhijian, Lu Guiming, A Novel Semantic Web Service Composition Algorithm Based on QoS Ontology, 2010 IEEE International Conference on Computer and Communication Technologies in Agriculture Engineering, pg. 166-168 
[6] Hongjiang Cao, Guihua Nie, Donglin Chen, Research on the Web Service Composition Based on Semantic, 2010 2nd IEEE International Conference on Computer Engineering and Technology ,pg770-773.

[7] Peter Bartalos and M’aria Bielikov'a, QoS Aware Semantic Web Service Composition Approach Considering Pre/Postconditions, 2010 IEEE International Conference on Web Services, pg.345-352.

[8] Yan Lu, Zhaozi Gao, Kai Chen, A Dynamic Composition Algorithm of Semantic Web Service Based on QoS, 2010 IEEE Second International Conference on Future Networks,pg 354-356.

[9] Anne H.H. Ngu, Michael P. Carlson, Quan Z. Sheng, , Semantic-Based Mashupof Composite Applications, IEEE Transactions On Services Computing, Vol. 3, No. 1, January-March 2010,pg.2-15

[10] David Chiu, Travis Hall, Farhana Kabir, An Approach towards Automatic Workflow Composition, IDEAS11 2011, September 21-23, Lisbon [Portugal], Copyright 2011 ACM 978-1-4503-0627$0 / 11 / 09$

[11] Howard Gartner, http://www.indiana.edu/ intell/gardner.shtml, January 26, 2012

[12] http://oxforddictionaries.com/definition/english/intelligence

[13] Rashmi Bansal, Stay Hungry, Stay Foolish [14] Richard
http://www.lehman.edu/deanhum/philosophy/platofootnote/PlatoFootnote.org/Talks_files/race.pdf

[15] David Goleman, https://www.edonline.sk.ca/bbcswebdav/library/curricula/English/Social_Studies/Psychology_20_200 2.pdf

[16] http://en.wikipedia.org/wiki/Tencent_Holdings

[17] Grigoria Antoniou, Frank Van Harmelen, The Semantic Web Primer, MIT Press, 2004

[18] Ian Horroks, Markus Krötzsch, František Simancík , A description logic primer, http://arxiv.org/pdf/1201.4089.pdf

\section{AUTHORS}

P. Sandhya is a research scholar in Faculty of Computer Science and Assistant Professor in the Department of Information Technology. She is interested in Semantic Web Services.

Dr. M Lakshmi is currently working as Professor and Head of Department of Computer Science and Engineering in Sathyabama University, Chennai. She has an experience of more than 15 years in Teaching and working in

Sathyabama University from 1995 holding different positions. She is currently engaged in a cumulative research project funded by Indira Gandhi Center for Atomic Research (IGCAR), Kalpakkam

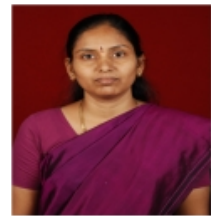

